\title{
A COMPLETE BOOLEAN ALGEBRA OF SUBSPACES WHICH IS NOT REFLEXIVE
}

\author{
BY JOHN B. CONWAY \\ Communicated by Paul R. Halmos, December 27, 1972
}

This note provides a negative answer to a question raised by P. R. Halmos [2, Problem 9]. For the convenience of the reader, the terminology necessary to understand the question is presented here. Let $\mathscr{L}$ be a lattice of subspaces of a Hilbert space $\mathscr{H}$ and let Alg $\mathscr{L}$ be the algebra of all bounded operators in $\mathscr{B}(\mathscr{H})$ that leave each subspace in $\mathscr{L}$ invariant. If $\mathscr{A} \subset \mathscr{B}(\mathscr{H})$, let Lat $\mathscr{A}$ be the lattice of all subspaces of $\mathscr{H}$ that are left invariant by each operator in $\mathscr{A}$. A lattice $\mathscr{L}$ is reflexive if Lat $\mathrm{Alg} \mathscr{L}=\mathscr{L}$. If $\mathscr{L}$ is a reflexive lattice and $\left\{P_{i}\right\}$ is a net of orthogonal projections such that $P_{i}(\mathscr{H}) \in \mathscr{L}$ for each $i$ and $P_{i} \rightarrow P$ in the strong operator topology then $P(\mathscr{H}) \in \mathscr{L}$; in other words, $\mathscr{L}$ is strongly closed. It is true that a strongly closed lattice of subspaces is a complete lattice, but the converse is false.

A Boolean algebra of subspaces is a distributive lattice $\mathscr{L}$ such that for each $M$ in $\mathscr{L}$ there is a unique $M^{\prime}$ in $\mathscr{L}$ such that $M \cap M^{\prime}=(0)$ and $M \vee M^{\prime} \equiv\left(M+M^{\prime}\right)^{-}=\mathscr{H}$. (Note that it is only required that $\mathscr{H}$ be the closure of $M+M^{\prime}$.) Problem 9 of [2] asks: Is every complete Boolean algebra of subspaces reflexive? The answer is no, and this is shown in this paper by giving a complete Boolean algebra of subspaces which is not strongly closed. In one sense this answer seems unsatisfactory because a new question arises: Is every strongly closed Boolean algebra of subspaces reflexive? In another sense the answer is satisfying because the original question was the proper one to ask. The property of completeness is a lattice theoretic one, while the property of being strongly closed is not.

For the remaining terminology the reader is referred to [4] and other standard references. If $X=[0,2 \pi]$, let $\mu$ be a positive singular measure on the collection $\mathscr{A}$ of Borel subsets of $X$. For $A$ in $\mathscr{A}$ define

$$
\varphi_{A}(z)=\exp \left(-\int_{A} \frac{e^{i \theta}+z}{e^{i \theta}-z} d \mu(\theta)\right), \quad|z|<1,
$$

and put $\varphi=\varphi_{X}$. Each $\varphi_{A}$ is an inner function, and $\varphi_{A}$ is a divisor of $\varphi_{B}$ if and only if $A \subset B$. $\mathscr{H}=H^{2} \ominus \varphi H^{2}$ and, for each $A$ in $\mathscr{A}, M_{A}=\varphi_{A} H^{2}$ $\Theta \varphi H^{2}$.

AMS (MOS) subject classifications (1970). Primary 47A15; Secondary 06A40, 46L15. 


$$
M_{A} \cap M_{B}=M_{A \cup B} .
$$

In fact, $\varphi_{A} H^{2} \cap \varphi_{B} H^{2}=\psi H^{2}$ where $\psi$ is the least common multiple of $\varphi_{A}$ and $\varphi_{B}$. It is easy to check that $\psi=\varphi_{A \cup B}$ and from this it follows that (1) holds. Similarly

$$
M_{A} \vee M_{B}=M_{A \cap B} .
$$

It follows from (1) and (2) that $\mathscr{L}=\left\{M_{A}: A \in \mathscr{A}\right\}$ is a distributive lattice; and, with $M_{A}^{\prime}=M_{X-A}, \mathscr{L}$ is a Boolean algebra of subspaces of $\mathscr{H}$.

LeMMA. $\mathscr{L}=\left\{M_{A}: A \in \mathscr{A}\right\}$ is a complete Boolean algebra.

Proof. It suffices to show that if $\mathscr{B} \subset \mathscr{A}$ then there is an $A$ in $\mathscr{A}$ with $M_{A}=\bigcap\left\{M_{B}: B \in \mathscr{B}\right\}$. Because of $(1), \mathscr{B}$ may be assumed to be closed under finite unions. If $\beta=\sup \{\mu(B): B \in \mathscr{B}\}$ then there is an increasing sequence $\left\{B_{n}\right\}$ in $\mathscr{B}$ such that $\beta=\lim _{n} \mu\left(B_{n}\right)$. If $A=\bigcup\left\{B_{n}: n \geqq 1\right\}$ then $\mu(A)=\beta$ and $\mu(B-A)=0$ for every $B$ in $\mathscr{B}$. It is claimed that $\varphi_{A}=$ 1.c.m. $\left\{\varphi_{B}: B \in \mathscr{B}\right\}$. In fact, if $B \in \mathscr{B}$ then $\varphi_{A}=\varphi_{A-B} \varphi_{B}$ since $\mu(B-A)=0$. Also, if $\psi$ is an inner function that is a multiple of $\varphi_{B}$ for each $B$ in $\mathscr{B}$ then, for every integer $n, \psi=\varphi_{B_{n}} \psi_{n}$ for some inner function $\psi_{n}$. But $\varphi_{B_{n}}(z) \rightarrow$ $\varphi_{A}(z)$ for every $z$ so it follows that $\psi_{n}(z) \rightarrow \Psi(z)$ for some inner function $\tilde{\psi}$. Hence $\psi=\varphi_{A} \Psi$ and $\varphi_{A}=1$. c.m. $\left\{\varphi_{B}: B \in \mathscr{B}\right\}$. Consequently,

$$
M_{A}=\bigcap\left\{M_{B}: B \in \mathscr{B}\right\} .
$$

THEOREM. $\mathscr{L}=\left\{M_{A}: A \in \mathscr{A}\right\}$ is reflexive if and only if $\mu$ is a purely atomic measure.

Proof. If $\mu$ is purely atomic then $\mathscr{L}$ is an atomic Boolean algebra and hence is reflexive [3]. To prove the converse, two additional results are needed. The first of these can be found in [5] although the proof contains an error (which can be rectified). However, in the case under consideration (where $L^{1}(\mu)$ is separable) the proof is valid. (Also see [1].)

TheOREM A. Let $(X, \mathscr{A}, \mu)$ be a decomposable nonatomic measure space and let $f \in L^{\infty}(X, \mathscr{A}, \mu)$ such that $0 \leqq f \leqq 1$. Then there is a sequence $\left\{A_{n}\right\}$ in $\mathscr{A}$ such that $\chi_{A_{n}} \rightarrow f$ in the weak-star topology of $L^{\infty}$.

THEOREM B. For each inner function $q$ let $E_{q}$ be the orthogonal projection of $\mathrm{H}^{2}$ onto $q \mathrm{H}^{2}$. If $q, q_{1}, q_{2}, \ldots$ are inner functions such that $q(z)$ $=\lim _{n} q_{n}(z)$ for $|z|<1$ then $E_{q_{n}} \rightarrow E_{q}$ strongly.

PRoof. If $z^{m}$ is the function that assumes the value $a^{m}$ at $a$ then it is easily verified that 


$$
E_{q}\left(z^{m}\right)=q \sum_{k=0}^{m} \frac{1}{k !} \overline{q^{(k)}(0)} z^{m-k}
$$

It follows that $E_{q}\left(z^{m}\right)(a)=\lim _{n} E_{q_{n}}\left(z^{m}\right)(a)$ for $|a|<1$. This gives that $E_{q_{n}}\left(z^{m}\right) \rightarrow E_{q}\left(z^{m}\right)$ weakly in $H^{2}$. Since polynomials are dense in $H^{2}, E_{q_{n}} \rightarrow E_{q}$ in the weak operator topology. But for projections weak convergence is equivalent to strong convergence, and the proof is complete.

Suppose $\mu$ is not purely atomic; the proof of the main theorem will be completed by showing that $\mathscr{L}$ is not strongly closed. There is a set $A$ in $\mathscr{A}$ that contains no atoms for $\mu$ and with $\mu(A)>0$. Let $f$ be any Borel function such that $0 \leqq f \leqq 1, f(x)=0$ for $x$ in $X-A$, and $0<f(x)<1$ on a set of positive measure. According to Theorem A there is a sequence $\left\{A_{n}\right\}$ in $\mathscr{A}$ such that $A_{n} \subset A$ and $\chi_{A_{n}} \rightarrow f$ in the weak-star topology of $L^{\infty}(\mu)$. For each $z,|z|<1$,

$$
\varphi_{A_{n}}(z) \rightarrow \psi(z)=\exp \left(-\int \frac{e^{i \theta}+z}{e^{i \theta}-z} f(\theta) d \mu(\theta)\right) .
$$

Theorem B implies that $E_{\varphi_{A_{n}}} \rightarrow E_{\psi}$ strongly; so $E_{\varphi_{A_{n}}}-E_{\varphi} \rightarrow E_{\psi}-E_{\varphi}$ strongly. It is straightforward to show that if $P_{A}$ is the projection of $\mathscr{H}$ onto $M_{A}$, then $P_{A_{n}} \rightarrow P_{\psi}$, where $P_{\psi}$ is the projection of $\mathscr{H}$ onto $\psi H^{2} \ominus \varphi H^{2}$. Since $\psi H^{2} \ominus \varphi H^{2} \neq M_{A}$ for any $A$, the proof is complete.

Finally, it should be pointed out that $\mathscr{L}$ is isomorphic to the reflexive Boolean algebra Lat $T$, where $T$ is multiplication by the independent variable on $L^{2}(X, \mu)$.

\section{REFERENCES}

1. J. B. Conway and J. Szücs, The weak sequential closure of certain sets of extreme points in a von Neumann algebra, Indiana Univ. Math. J. 22 (1973), 763-768.

2. P. R. Halmos, Ten problems in Hilbert space, Bull. Amer. Math. Soc. 76 (1970), 887-933. MR 42 \# 5066.

3. $\longrightarrow$, Reflexive lattices of subspaces. J. London Math. Soc. (2) 4 (1971), 257-263. MR 44 \# 5808.

4. K. Hoffman, Banach spaces of analytic functions, Prentice-Hall, Englewood Cliffs, N.J., 1962. MR 24 \# A2844.

5. R. B. Holmes, A geometric characterization of nonatomic measure spaces, Math. Ann. 182 (1969), 55-59. MR 40 \# 1571.

Department of Mathematics, Indiana University, Bloomington, Indiana 47401 